\title{
VARIATIONS INTRASPECIFIQUES CHEZ UN CARMYERIUS (TREMATODA GASTROTHYLACIDAE) PARASITE DE BUFFLE DU CONGO BELGE
}

\author{
Par Robert-Ph. DOLLFUS
}

Lors de l'étude d'une collection de Paramphistomes récoltés au Congo belge, chez des Bos (Syncerus) caffer Sparmann, j’ai réuni, sous le nom de Carmyerius endopapillatus $\mathrm{n}$. sp., des spécimens provenant de diverses localités et présentant entre eux des différences bien nettes, mais qui ne m'ont pas paru assez importantes pour caractériser plusieurs espèces séparées. Ces spécimens ont pu être répartis en trois formes que je désigne ici comme formes $\mathrm{A}, \mathrm{B}$ et $\mathrm{C}$.

\section{FORME A (fig. 1-3)}

Matériel de la collection. - Le tube $\mathrm{n}^{\circ}$ 3777-3934, étiqueté * Van Risseghem, dans bovidés; Kasaï: Tshikapa » contient environ une cinquantaine de Paramphistomes, mélange de nombreux Cotylophoron et de quelques Carmyerius. Ces derniers sont, soit sub-pisiformes $(5 \times 4 \mathrm{~mm}$. ou diamètre env. $4 \mathrm{~mm}$.), soit piriformes (longueur 5,5-6 mm., largeur $3 \mathrm{~mm}$.).

Je suppose que les * bovidés » ayant fourni ces matériaux étaient des Bos caffer Sparmann.

La description ci-après concerne seulement les spécimens piriformes mesurant $5,5 \times 3 \mathrm{~mm}$.

La section transversale est à peu près circulaire. La surface de la cuticule porte de petites papilles dans la partie avoisinant l'orifice buccal et l'ouverture en fente transversale de la poche ventrale; ces papilles sont plus ou moins apparentes selon les individus.

L'acétabulum, très profond, plus ou moins sphérique (diam. 1,5-1,7 mm.), s'ouvre à l'extrémité postérieure, dans l'axe longitudinal du corps.

La poche ventrale a une paroi épaisse comme le corps ; elle est très réduite ; elle a, antérieurement, à peu près la forme d'un demi-cercle, avec courbure vers la face ventrale ; plus postérieurement, elle est subcirculaire puis se rétrécit et se termine ventralement à l'acétabulum, vers la mi-hauteur de celui-ci. La paroi interne de la poche ventrale est couverte dans sa partie antérieure de papilles coniques, latéralement et dorsalement. Celles-ci s'étendent jusqu'à l'ouverture externe de la poche et ne s'étendent pas, ou à peine, postérieurement à l'atrium génital. 
L'orifice buccal est très petit ; un très court prépharynx aboutit au pharynx, subsphérique, avec un diamètre de 0,57 à $0,58 \mathrm{~mm}$.

L'œsophage a une paroi très épaisse, avec un diamètre de $0,20-0,23$, sa longueur est au moins égale au pharynx et peut atteindre 0,7 ; elle varie quelque peu avec l'extension de la région antérieure.

Les deux cæca divergent presque à angle droit puis se recourbent, décrivent des sinuosités et atteignent le bord antérieur des testicules. Ceux-ci sont très volumineux, massifs, à peine un peu lobés et mesurent, en moyenne, $1,4 \times 1,5$;

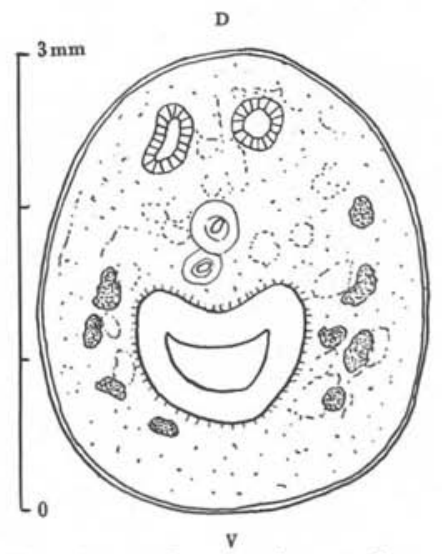

Fig. 1. - Carmyerius endopapillatus n. sp. forme A. Coupe transversale un peu postérieure au niveau de l'atrium génital, pour monirer la forme de la poche ventrale.

ils sont au contact de l'acétabulum et presque entièrement en avant de lui ; leur extrémité postérieure seule est au niveau du fond de la cavité acétabulaire ; ils se touchent dans le plan sagittal. Le canal déférent devient musculeux et ensuite pars prostatica avec un diamètre de 0,23-0,27 pour aboutir au pénis.

L'ovaire, très petit, est entre les testicules et la paroi dorsale.

L'utérus est volumineux; il s'étend postérieurement jusqu'à l'acétabulum, passant dans le petit espace triangulaire médian, laissé libre entre les testicules et la paroi dorsale du corps.

Les œufs mesurent, chez un même individu: $120 \times 78,126 \times 79,128 \times$ $76,132 \times 76,132 \times 78 \mu$.

Les vitellogènes sont latéraux, ventraux et dorsaux, dispersés très irrégulièrement entre le niveau de la bifurcation intestinale et l'acétabulum.

Le pore génital est situé au niveau de l'œsophage, en avant de la bifurcation intestinale, dans une dépression de la paroi dorsale de la poche ventrale. Il est 


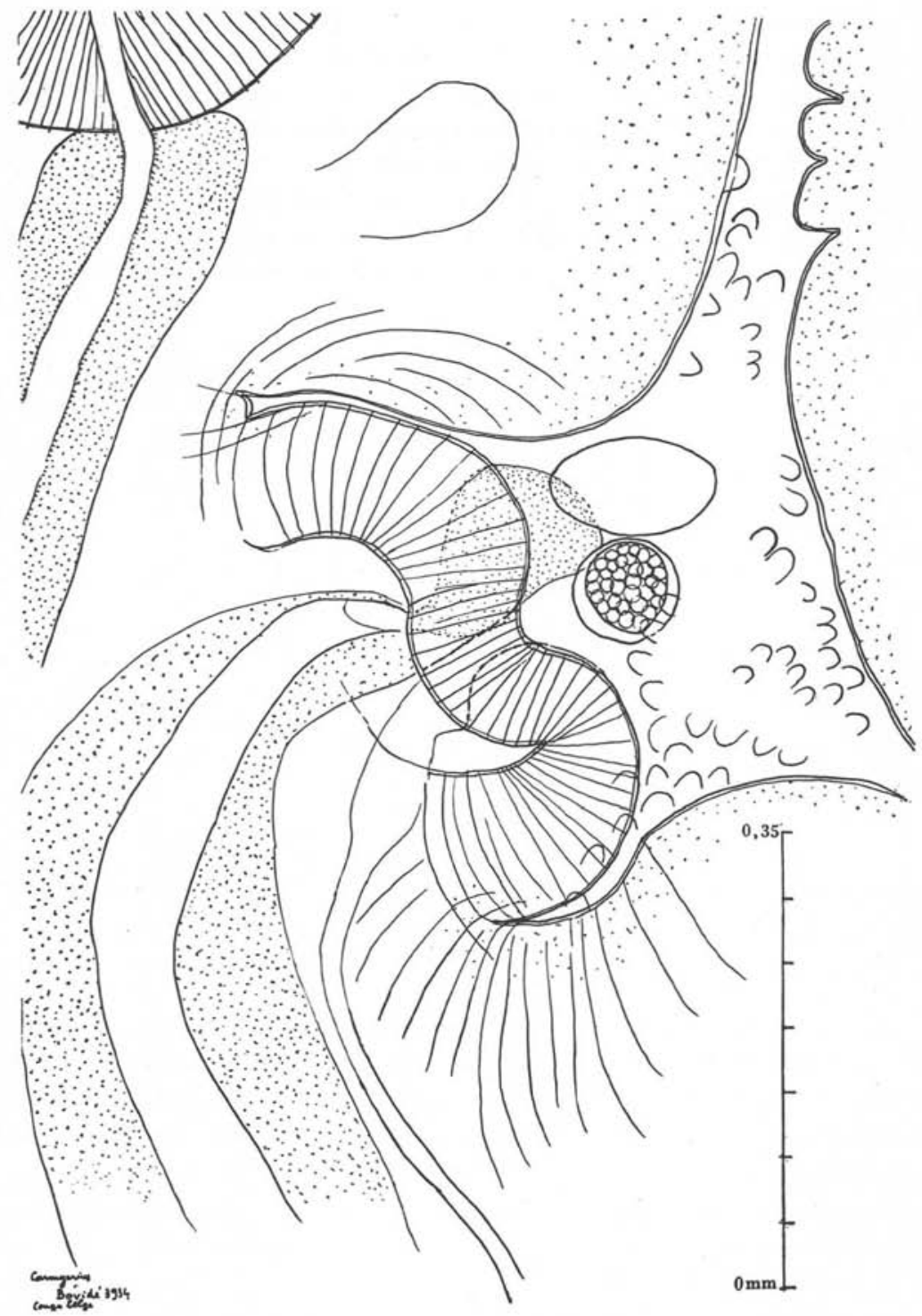

FIG. 2. - Musculature entourant le pore génital. Coupe à peu près sagittale

entouré par une forte musculature bien développée, formant un anneau délimité ventralement et dorsalement mais qui, à sa périphérie, n'est pas délimité dorsalement du parenchyme environnant; ce n'est donc pas un vrai sphincter. 
Toute la partie atriale de la poche ventrale est garnie de papilles coniques et ce revêtement de papillés s'étend jusqu'à l'ouverture externe de la poche, rejoignant le revêtement de papilles de l'extrémité antérieure du corps.

Discussion. - Deux caractères sont particulièrement importants à considérer : $1^{\circ}$ la présence de papilles dans la partie antérieure, atriale, de la poche ventrale ; $2^{\circ}$ la présence d'une forte musculature entourant le pore génital.

A ma connaissance, des papilles dans la dépression atriale de la poche ventrale ne sont connues que chez Carmyerius wenyoni (R. T. Leiper), de l'estomac de Onotragus mariae Gray, de Taufikia (Nil blanc). R. T. Leiper (1908, p. 197) dit ceci : « The genital pore lies at the bottom of a distinct depression,

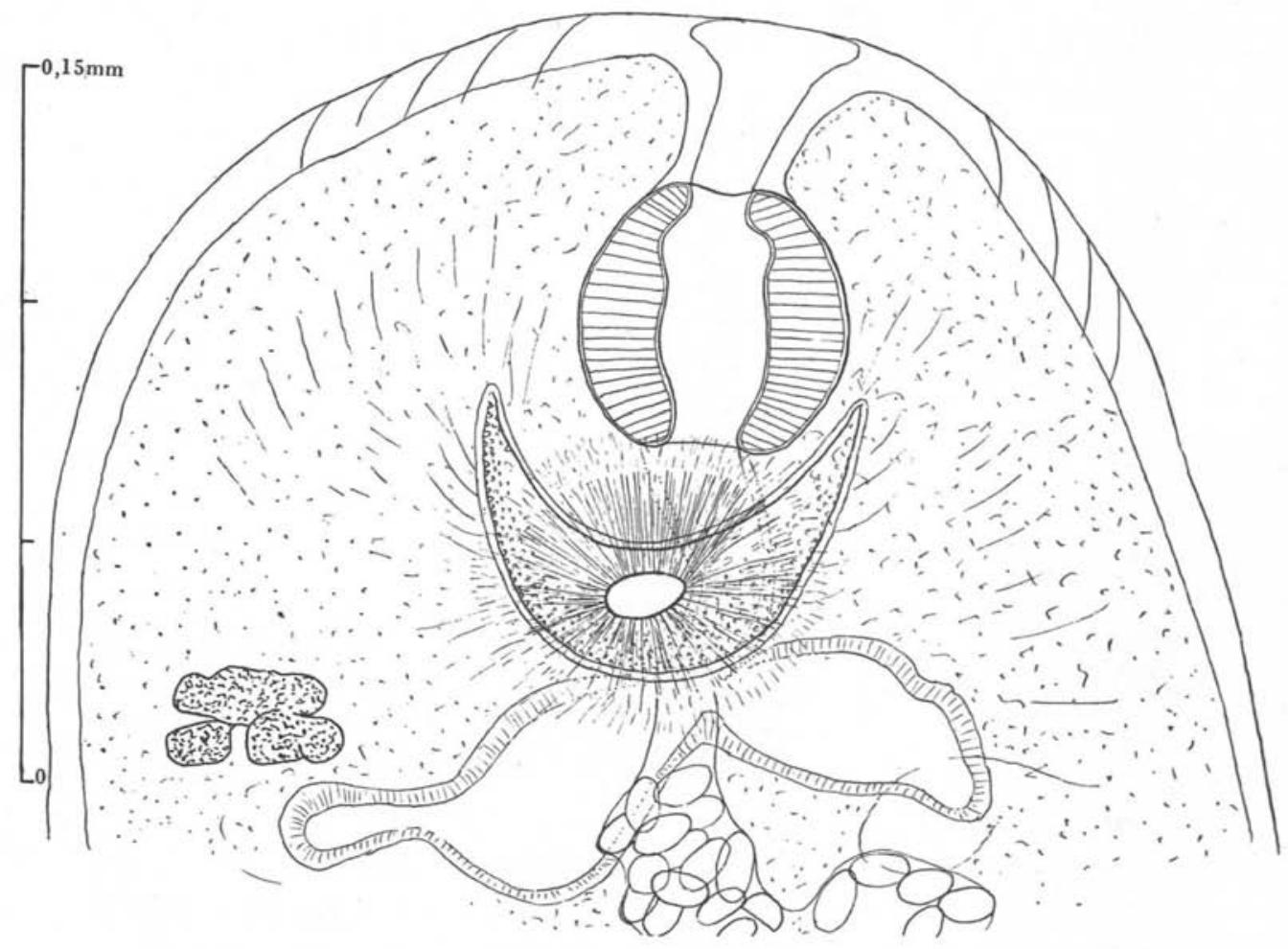

Fig. 3. - Partie dorsale de la région antérieure d'un individu sectionné suivant un plan frontal. Toute la poche ventrale a été enlevée, sauf le diverticule atrial de la poche. Ce diverticule, sur cette coupe, a une forme de croissant. Par cette ouverture, on voit l'orifice génital entouré de sa musculature et les papilles des parois de la poche ventrale. L'oesophage et la bifurcation intestinale sont dans un plan plus profond. L'utérus passe dans un plan intermédiaire, ventralement à la bifurcation intestinale. L'appareil terminal ơ n'est pas figuré. A gauche, un groupe de follicules vitellogènes. 
the sides of which are covered with papillae. ». Chez wenyoni, il n'y a pas de musculature particulièrement développée autour du pore génital, l'emplacement du pore génital est différent, la forme de la poche ventrale est différente ; notre espèce du Congo belge n'est donc pas référable à wenyoni.

Une musculature atriale particulière est connue chez $C$. synethes (F. Fischoeder 1901), C. graberi S. Grétillat 1960, C. dollfusi Y. Golvan, A. Chabaud et S. Grétillat 1957, mais il n'existe pas de papilles atriales chez ces espèces et elles diffèrent par beaucoup d'autres caractères de celle ci-dessus décrite; j'estime donc que cette dernière est nouvelle.

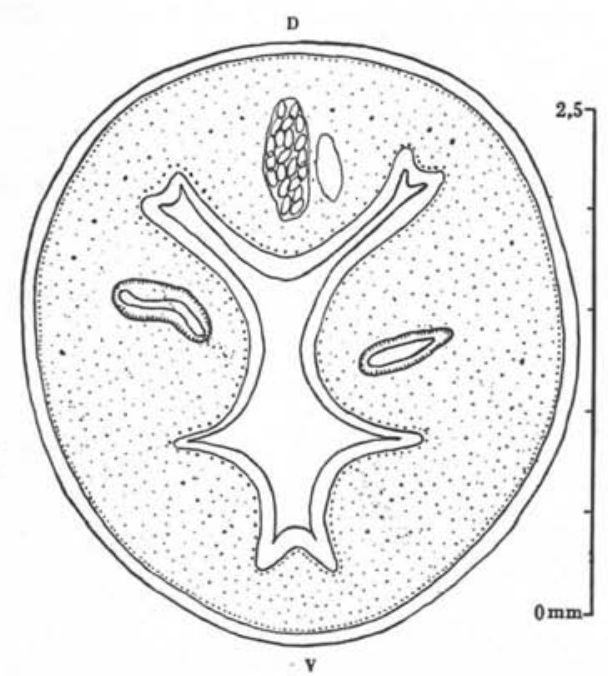

FIG. 4. - Carmyerius endopapillatus mihi, forme B. Section de la poche ventrale en arrière de l'atrium génital (spécimen du tube 87).

FORME B (fig. 4-8)

Matériel de la collection. - Trois tubes, $\mathrm{n}^{\circ \times} 85,86,87$, étiquetés : * estomac de Bos caffer - Menzadi - D ${ }^{r}$ E. Dartevelle, avril 1937 ».

- Le tube 85 contient environ 76 Paramphistomes, mélange de Carmyerius et de Paramphistomum ou Cotylophoron, qui n'ont pas été examinés. Environ 10 Carmyerius ont été prélevés pour étude.

Des individus atteignant $6,5 \times 3 \mathrm{~mm}$. sont encore immatures.

- Le tube 86 contient environ 44 Paramphistomes, mélange de Carmyerius et Paramphistomum ou Cotylophoron, qui n'ont pas été examinés. 4 Carmyerius ont été prélevés pour étude. 
- Le tube 87 contient environ 125 Paramphistomes, 70 d'entre eux ont été séparés ; parmi eux, il y avait environ 58 Paramphistomum ou Cotylophoron, qui n'ont pas été examinés, et 12 Carmyerius qui ont été prélevés pour étude.

Description (sauf indication contraire, d'après des spécimens du tube 87 ). - Les spécimens mesurent de $4 \times 2,5 \mathrm{~mm}$. à $9,6 \times 5 \mathrm{~mm}$., mais la plupart ont une longueur ne dépassant pas 6 à $7 \mathrm{~mm}$. et une largeur de 3,5 à $4 \mathrm{~mm}$. La forme générale est subpiriforme, la section transversale est plus ou moins circulaire à tous les niveaux. La cuticule présente de fines striations transver-

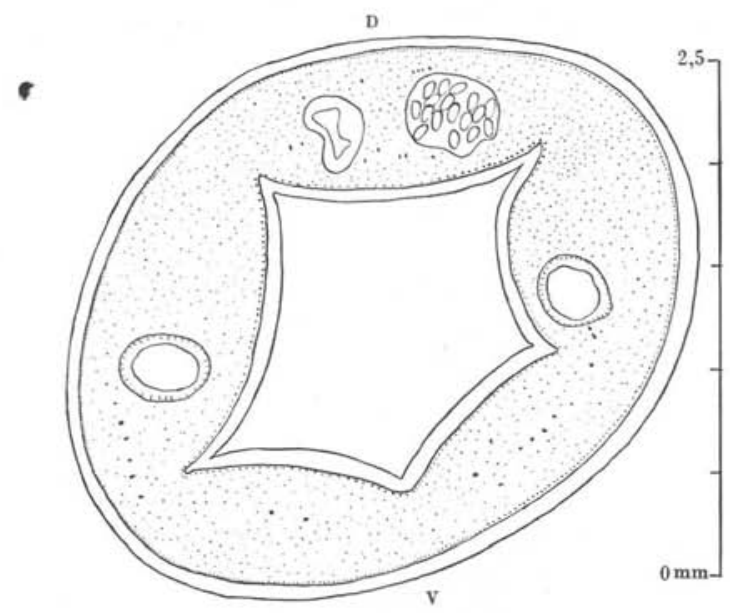

Fig. 5. - Id. chez un autre individu (spécimen du tube 87)

sales-circulaires; elle est antérieurement ornée de papilles ; le revêtement de papilles ne s'éténd qué très peu en arrière de l'ouverture en fente transversale de la poche ventrale, mais se continue dans la poche ventrale. L'acétabulum a son orifice fortement festonné et s'ouvre dans l'axe longitudinal du corps ; pour un spécimen mesurant $6,2 \times 3,6 \mathrm{~mm}$., l'acétabulum mesure longitudinalement 1,276 et transversalement $1,738 \mathrm{~mm}$.

La poche ventrale, à paroi épaisse, est en forme de fente allongée dorsoventralement, subpentagonale, avec un côté dorsal et l'angle opposé ventral (fig. 4, 5). Des figures de coupes transversales données par P. A. Maplestone (1923, p. 188-189, fig. 23-24) pour Carmyerius exoporus Maplestone montrent des formes de section de poche ventrale très voisines de celles de mes figures 4-5, mais mes spécimens congolais n'appartiennent évidemment pas à exoporus, le pore génital étant dans l'intérieur de la poche. Chez les individus des tubes 85 et 86 , la section transversale de la poche ventrale est un peu différente (fig. 6 et 7). Dans sa partie antérieure, la paroi de la poche est garnie de papil- 
les coniques, serrées, qui ne s'étendent que peu en arrière de l'atrium génital. Dans ses parties moyenne et postérieure, la paroi interne de la poche montre souvent une annulation transversale. L'entonnoir buccal est très court, environ 120-125 $\mu$. Pour un individu mesurant $6,2 \times 3,6 \mathrm{~mm}$., le pharynx a une lon-

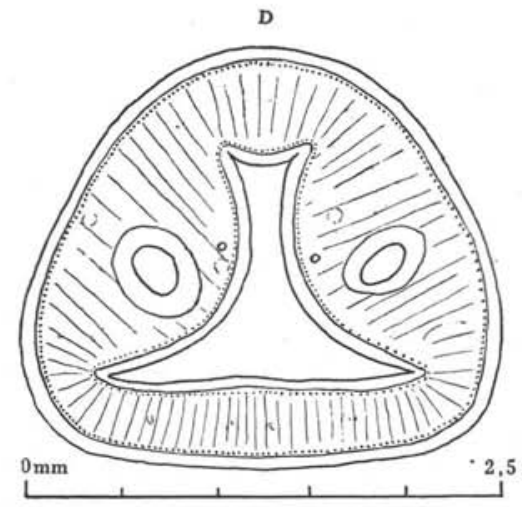

Fig. 6. - Section de la poche ventrale chez un spécimen du tube 85

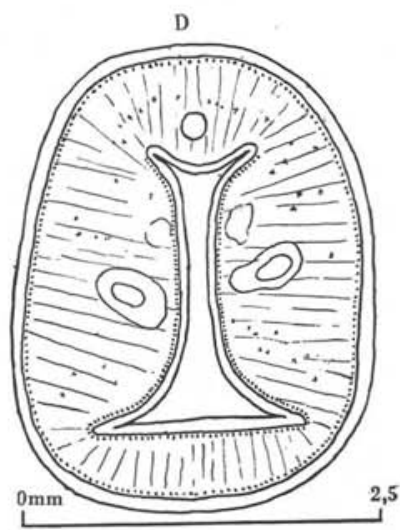

Fig. 7. - Section de la poche ventrale chez un spécimen du tube 86 .

gueur de $445 \mu$, une largeur de $384 \mu$; l'œsophage à paroi épaisse est long d'environ 0,46 , avec un diamètre d'environ 0,123 . Les cæca sont un peu sinueux et se terminent souvent sans atteindre le bord antérieur des testicules; ils s'étendent rarement jusqu'à ce niveau.

Les testicules sont volumineux, non lobés, entièrement en avant de l'acétabulum, ou presque, et mesurent, par exemple, longitudinalement 1,076 et transversalement $1,261 \mathrm{~mm}$. 


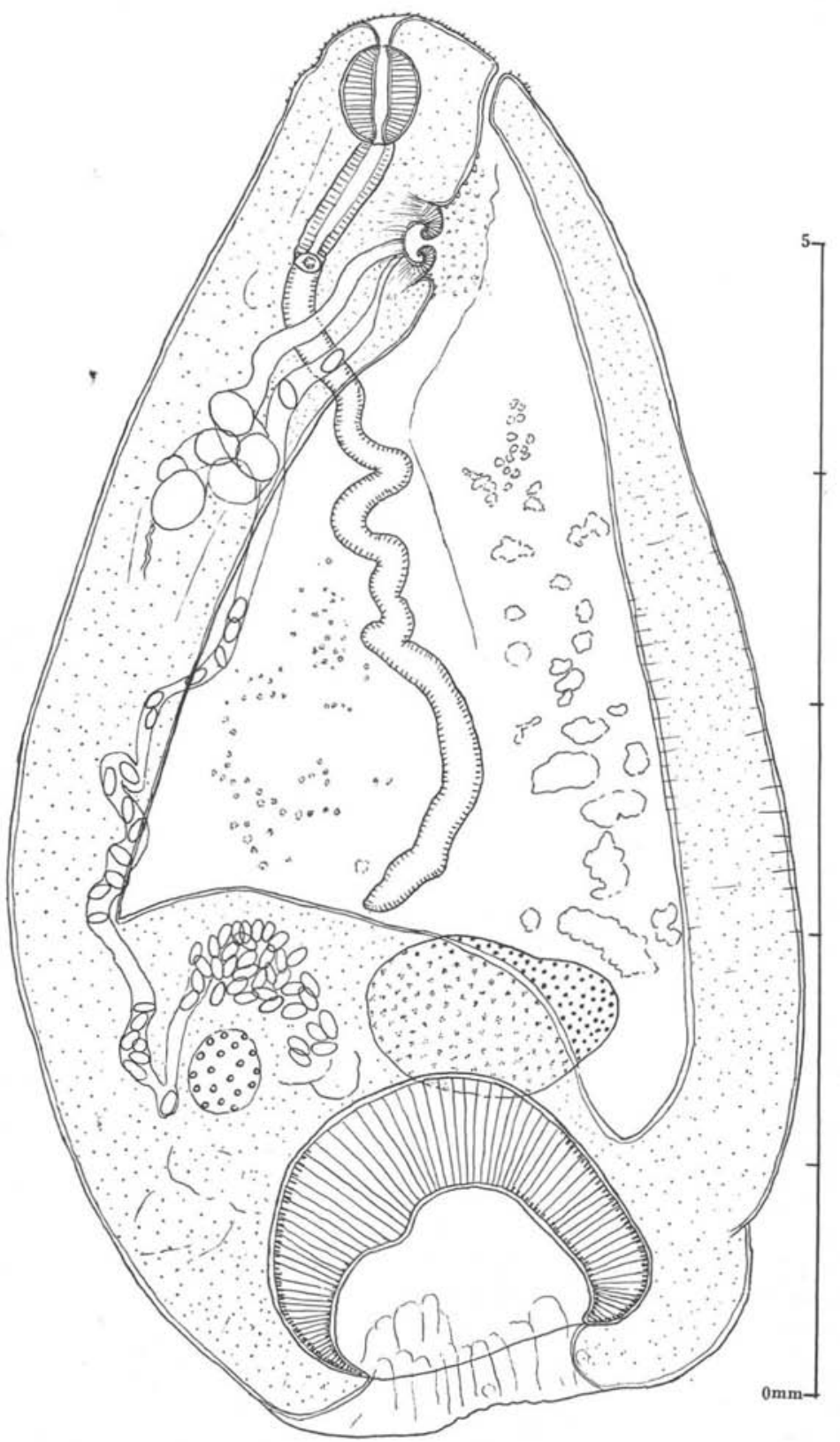

Fig. 8. - Partie gauche d'un individu coupé sagittalement 
L'ovaire est petit, globuleux, avec un diamètre de 0,40-0,48 mm., tantôt au contact de l'acétabulum, tantôt un peu en avant. L'utérus atteint presque, postérieurement, l'acétabulum, il s'étend ventralement à la vésicule séminale, très contournée et dorsalement aux cæca, puis passe entre ceux-ci en direction antéro-ventrale pour atteindre le pore génital. Les œufs mesurent, chez un

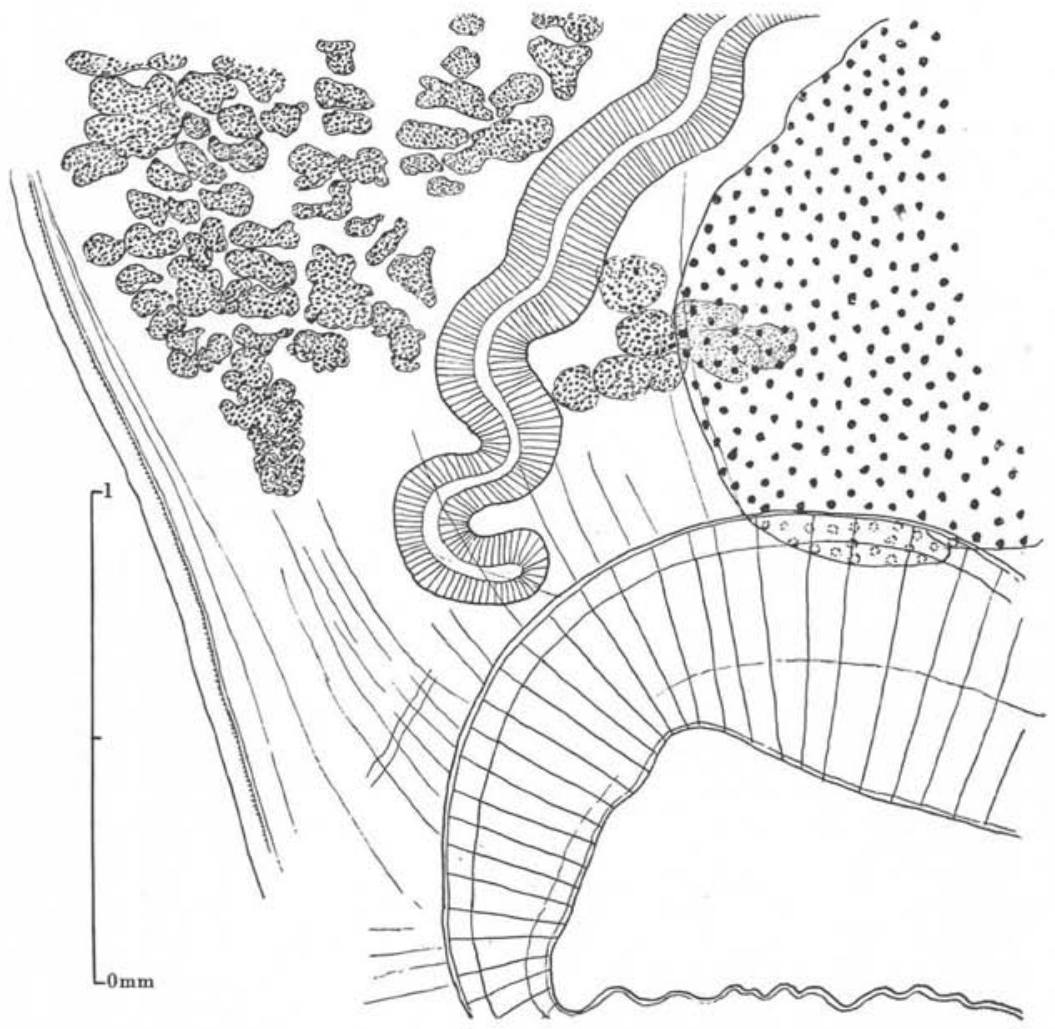

Fıg. 9. - Carmgerius endopapillatus mihi, forme C. Terminaisôn du caecum droit vers le niveau du bord antérieur de l'acétabulum, ventralement. Coupe sagittale épaisse. Côté droit (spécimen du tube $\mathrm{n}^{\circ} 6544$ ).

même individu, $132-140 \times 77 \mu$ à $145,7 \times 74,2 \mu$. La maturité génitale est indépendante de la taille des individus, un spécimen mesurant $7 \times 4 \mathrm{~mm}$. n'a pas commencé à produire d'œufs. Les vitellogènes sont très peu développés ; quelques follicules sont dispersés latéralement et ventralement en avant de l'acétabulum, dans le tiers moyen de la longueur du corps.

Le pore génital est situé au niveau de l'œsophage ou de sa bifurcation, il est entouré d'une musculature bien développée, formant un anneau délimité 
ventralement et dorsalement, mais qui, à sa périphérie, n'est pas délimité dorsalement du parenchyme environnant. L'atrium et la partie avoisinante de la poche ventrale sont garnis de papilles coniques serrées et ce revêtement de papilles rejoint celui de la cuticulé éxtérne apicale.

Discussion. - La forme ci-dessus décrite diffère à peine de la forme $\mathrm{A}$, décrite dans les pages précédentes. Cependant, il y a deux caractères qui per-

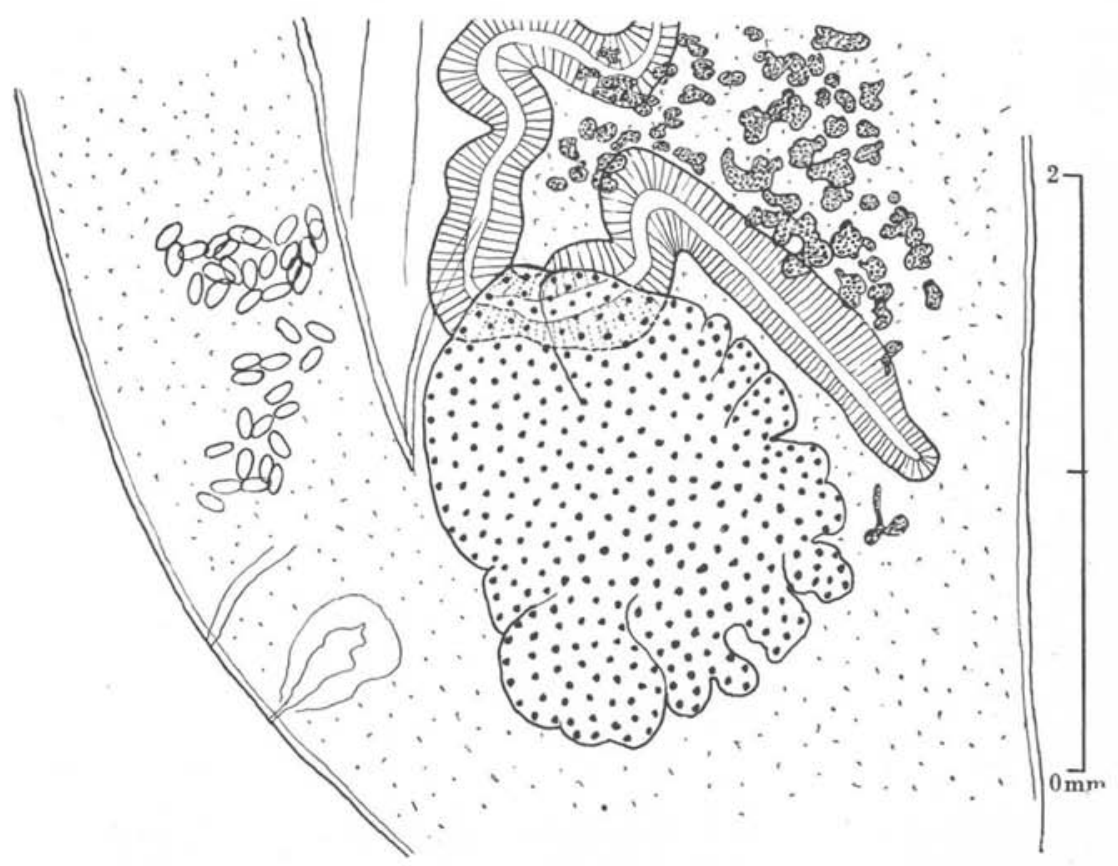

FIG. 10. - Carmyerius endopapillatus mihi, forme C. Terminaison du cacum gauche vers le niveau de la mi-hauteur du testicule gauche, ventralement. Goupe épaisse presque parallèle au plan sagittal (Spécimen du tube $\mathrm{N}^{\circ} 6544$ ).

mettent de la distinguer : les cæca sont un peu plus courts, se terminant souvent sans atteindre le bord antérieur des testicules; la poche ventrale a une forme très nettement différente. S'agit-il de variations individuelles ? c'est possible, et peut-être qu'en sectionnant un grand nombre d'individus on trouverait des formes de passage. Etant dans l'incertitude, je considère les spécimens récoltés par Dartevelle à Menzadi comme une variation de la forme $\mathrm{A}$, récoltée par Van Risseghem à Tshikapa. 
FORME C (fig. 9-13)

Matériel de la collection. $-1^{\circ}$ Environ 50 individus dans un tube étiqueté * estomac de Bos caffer, $\mathrm{D}^{r} \mathrm{H}$. Schouteden, Kwamouth 5/1921, $\mathrm{n}^{\circ} 6492-$ 6544 ». $2^{\circ} 14$ individus dans un tube étiqueté * estomac de Bos caffer, $\mathrm{D}^{\mathrm{r}} \mathrm{H}$. Schouteden, Kwamouth, 5/1921, $\mathrm{n}^{\circ} 55$.

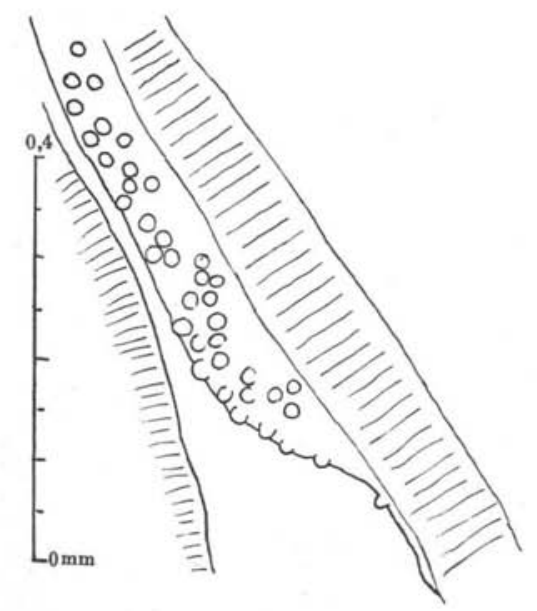

Fig. 11. - Id. forme C. Papilles sur le bord dorsal de la poche un peu en avant de la cavité atriale (spécimen du tube 55).

Description. - Corps piriforme, subcylindrique ou ovale, s'atténuant antérieurement. Section transversale subcirculaire à tous les niveaux; bouche et ouverture de l'acétabulum dans l'axe longitudinal du corps. Les dimensions varient de $5 \times 3$ à $9 \times 4,5 \mathrm{~mm}$. et $10 \times 4 \mathrm{~mm}$.

La cuticule de la région buccale porte de nombreuses petites papilles très pointues. L'entrée de l'acétabulum est plissée longitudinalement.

Pour un individu mesurant $8 \times 3 \mathrm{~mm}$., le pharynx est long de 1,046, large de 0,753 ; l'acétabulum mesure longitudinalement 1,292, transversalement $1,769 \mathrm{~mm}$. La poche ventrale varie de triangulaire avec angle ventral à pentagonale avec un sommet ventral et un côté dorsal. Sa paroi porte quelques grosses papilles sur le bord antérieur de la cavité atriale et plus en avant sur le bord dorsal de la poche. L'œsophage est un peu moins long que le pharynx, les cæca sont sinueux et atteignent soit le niveau du bord antérieur de l'acétabulum, soit le niveau du milieu des testicules, toujours ventralement aux testicules. 


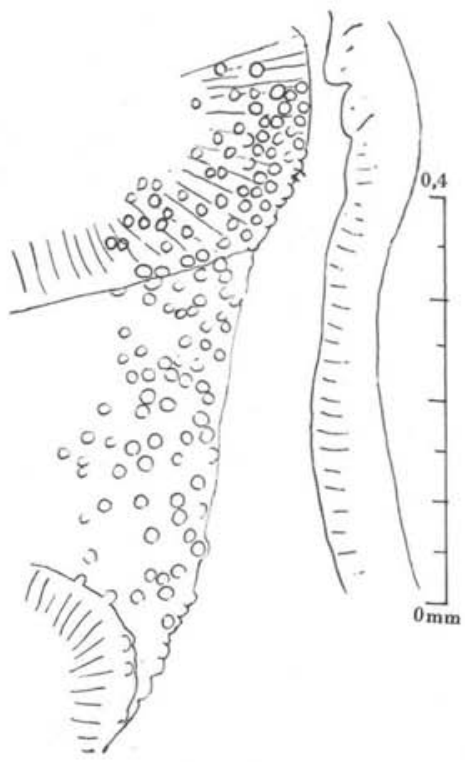

Fig. 12. - Id. forme C. Papilles sur le bord antérieur de la cavité atriale (spécimen du tube 55).

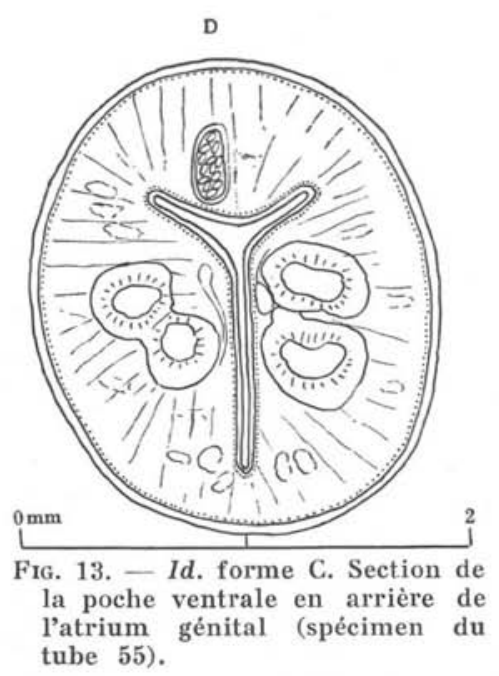

Les testicules sont gros, latéraux, un peu lobés, écartés ; ils peuvent être un peu plus longs que larges, par exemple $1,7 \times 1,3 \mathrm{~mm}$.

L'ovaire, relativement petit, est dorsal dans l'espace intertesticulaire. 
L'utérus, d'abord complètement dorsal, s'infléchit pour passer ventralement à la vésicule séminale, volumineuse et très contournée.

Les œufs mesurent $120-134,7 \times 66 \mu$.

Les vitellogènes sont constitués par de très nombreux petits follicules qui s'étendent latéralement et ventralement à la poche ventrale, atteignant antérieurement le niveau de l'œsophage et postérieurement le niveau de la mi-longueur des testicules.

Le pore génital est entouré d'une musculature particulière dont le diamètre atteint environ $610 \mu$. Il est situé au niveau de l'œsophage.

Discussion. - Cette forme $\mathrm{C}$ se distingue immédiatement de la précédente par la longueur des cæca se terminant ventralement aux testicules, les dépassant ou non. Les autres caractères distinctifs sont moins nets, comme le montre la comparaison des descriptions.

\section{Conclusion}

Des Carmyerius chez lesquels la dépression atriale de la poche ventrale est garnie de papilles ont été récoltés chez des Bos (Syncerus) caffer Sparmann de trois localités éloignées entre elles du Congo belge.

Pour chacune des trois localités, les spécimens récoltés présentent quelques caractères permettant de les distinguer de ceux des deux autres localités, mais les différences observées portant sur des caractères mineurs, l'ensemble des spécimens nous paraît devoir être considéré comme conspécifique, avec des variations dont l'amplitude est variable.

\section{BIBLIOGRAPHIE SOMMAIRE}

Brandes (Gustav P. H.), 1898. - Die Gattung Gastrothylax. Abhandl. Natur. forsch. Gesellschaft zu Halle, Bd XXI, Heft 1-3, 15-1-1898, p. 193$225+2$ p. ; pl. VII, fig. 1-16; pl. VIII, fig. 1-16.

Fischoeder (Franz), 1903. - Die Paramphistomiden der Säugethiere. Zoolog. Jahrbücher, System., Bd XVII, Heft 5-7, 9-2-1903, p. 485-660, fig. texte A-Q, pl. XX-XXXI, fig. 1-104.

Golvan (Yves), Сhabaud (Alain-G.) et Grétillat (Simon), 1957. - Carmyerius dollfusi n. sp. (Trematoda, Gastrothylacidae), parasite des Bovidés à

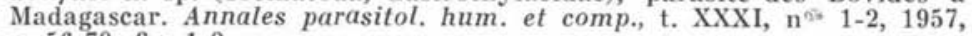
p. 56-70, fig. 1-9.

Grétrlllat (Simon), 1960. - Amphistomes (Trématodes) des ruminants domestiques de la République du Tehad. Description d'un Gastrothylacido nouveau Carmyerius graberi n. sp. Annales parasitol. hum. et comp., t. XXXV, n ${ }^{\circ} 4,1960$, p. 509-527, fig. 1-9C.

Leiper (Robert T.), 1908. - An account of some Helminthes contained in Dr C.M. Wenyon's Collection from the Sudan. $3 \mathrm{rd}$ Report Wellcome Research Laboratories at the Gordon Memorial College, Khartoum, 1908, p. 187-199, fig. texte 43-48C, pl. XXI, fig. 1-12 ; pl. XXIII, fig. 1-6.

Leiper (Robert T.), 1910. - The Entozoa of the Hippopotamus. Proceed Zoolog. Soc. London, febr. 1, 1910, p. 233-251, fig. 26-35.

Maplestone (P. A.), 1923. - A revision of the Amphistomala of Mammals. Annals Trop. Med. and Parasitol., Liverpool, vol. XVII, no 2, July 12, 1923 , p. 113-212, fig. texte 1-32, pl. V-VIII.

Stiles (Ch. Wardell) et Goldienger (Joseph), 1910. - A study of the anatomy of Watsonius (n. g.) watsoni of man... Hygienic Laboratory Bull., $\mathbf{n}^{\circ} 60$, Washington, April 1910, p. 1-259, fig. 1-205. 